\title{
Anticoagulation Therapy for Portal Vein Thrombosis in Patients with Cirrhosis in a Tertiary Center Experience
}

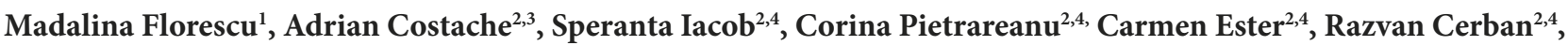 \\ Radu Dumitru' ${ }^{2,5}$, Mugur Grasu' ${ }^{2,5}$, Ioana Lupescu ${ }^{2,5}$, Liana Gheorghe ${ }^{2,4}$
}

1) Department of

Gastroenterology, Saint Marie

Clinical Hospital, Bucharest;

2) Carol Davila University

of Medicine and Pharmacy,

Faculty of Medicine,

Bucharest;

3) Dr. Ioan Cantacuzino

Clinical Hospital, Bucharest;

4) Centre for Digestive

Diseases and Liver

Transplantation, Fundeni

Clinical Institute, Bucharest;

5) Radiology and Imaging

Department, Fundeni Clinical

Institute, Bucharest,

Romania

\section{Address for correspondence: \\ Madalina Florescu \\ Saint Marie Clinical Hospital, \\ 37-39 Ion Mihalache \\ Boulevard, Bucharest, \\ Romania}

greere.madalina@gmail.com

\section{ABSTRACT}

Background \& Aims: The evidence regarding the use of anticoagulant (AC) agents in portal vein thrombosis (PVT) is increasing and, most patients undergo chronic treatment with low molecular weight heparin (LMWH) or vitamin K antagonists (VKA). Nevertheless, there are no clear data about who should receive antithrombotic therapy, when to initiate it, how long and what dose should be used for this set of patients. The aim of the study was to assess the outcome of patients with cirrhosis and portal vein thrombosis who received $\mathrm{AC}$ therapy, in terms of thrombus regression, bleeding events and survival rates.

Methods: This observational and retrospective study included 107 cirrhotic patients diagnosed with PVT in a single tertiary center between 2010-2019. 54 received low molecular weight heparin or vitamin K antagonist (AC treatment group) and 53 were untreated. All patients were periodically follow-up to assess the evolution of PVT (regression, progression, stable thrombus) and potential occurrence of bleeding events.

Results: The regression of portal vein thrombosis was significantly higher in the AC treatment group $(\mathrm{OR}=2.430 ; 95 \% \mathrm{CI}=1.11-6.167 ; \mathrm{p}=0.026)$, more than $50 \%$ of on-treatment patients experiencing regression of the thrombus. However, bleeding events were significantly more frequent in the AC treatment group (18.5\% vs. $7.5 \%)$ and the risk of bleeding was associated with thrombocytes less than 50x103/mm3 (OR=8.266; 95\%CI: $2.310-39.211 ; \mathrm{p}=0.002)$. Survival was better in the AC treatment group (68.4\% vs $48.7 \%$ at 5 years and $92.7 \%$ vs $77.8 \%$ at 1 year, $\mathrm{p}=0.038)$ and was lower in patients that experienced bleeding events $(37.22 \%$ survival at 5 years, mean time survival 44 months, $\mathrm{p}=0.008)$.

Conclusions: In our cohort of cirrhotic patients with PVT more than $50 \%$ of patients receiving AC therapy presented regression of the thrombus; most of them obtained partial recanalization. The bleeding complication rate was higher than expected, reaching $18 \%$. The overall mortality was lower in the treated group.

Key words: portal vein thrombosis - thrombus - anticoagulation therapy - cirrhosis - low molecular weight heparin - vitamin $\mathrm{K}$ antagonist - thrombocytes.

Abbreviations: AC: anticoagulant; CT: computed tomography; INR: international normalized ratio; LMWH: low molecular weight heparin; MELD: model for end stage liver disease; MRI: magnetic resonance imaging; PVT: portal vein thrombosis; VKA: vitamin $\mathrm{K}$ antagonists.

\section{INTRODUCTION}

Rare as it may be as a single pathophysiological entity, portal vein thrombosis (PVT) remains a common complication in cirrhotic patients. Multimodal imaging techniques together with periodic follow-up have led to early recognition of this vascular process. Portal vein thrombosis may have a negative impact on patient's outcome, increasing the portal hypertension, bleeding events and liver decompensation as well as reducing short term survival post liver transplantation [1]. The evidence regarding the use of anticoagulant (AC) agents in PVT is increasing and, most patients undergo chronic treatment with low molecular weight heparin (LMWH) or vitamin $\mathrm{K}$ antagonists (VKA) [2-4]. Furthermore, the state of "rebalanced hemostasis" which characterize most patients with liver cirrhosis, cannot predict the risk of bleeding when associated with AC therapy [5]. There are studies on the limited number of patients that promote the use of AC therapy for PVT regression; however, only a few of them demonstrated the use of AC with increased overall survival [6]. In addition to this, there are no clear data 
about who should receive antithrombotic therapy, when to initiate it, how long and what dose should be used for this set of patients. The main objective of this study was to assess the outcome of patients with cirrhosis and PVT who received AC therapy, in terms of thrombus regression, bleeding events and survival rates.

\section{METHODS}

This observational and retrospective study was conducted in a single tertiary center, Centre for Digestive Diseases and Liver Transplantation, Fundeni Clinical Institute, Bucharest, Romania. Data regarding adults with liver cirrhosis that had been diagnosed with nonmalignant PVT were researched using the Hippocrates network, between 2010-2019. Inclusion criteria were age 18 or above, end stage liver disease, presence of PVT, patients eligible for liver transplant. Exclusion criteria included hepatocellular carcinoma, Budd Chiari syndrome or patients not eligible for liver transplant. Demographic data consisted of age, gender, etiology of cirrhosis, severity and extension of PVT, Child-Pugh and model for end stage liver disease (MELD) scores. Portal vein thrombosis was defined as any echogenic mass formation in the portal vein lumen assessed by Doppler echography and confirmed by computer tomography (CT) or magnetic resonance (MRI) scans. After each data set had been collected, we divided our cohort into two groups: one that received AC therapy $(n=54)$ and one that did not $(n=53)$.

All patients underwent bleeding events prophylaxis: endoscopic screening was used and, according to the presence and severity of esophageal varices, the interventional (band ligation) or/and the pharmacological method (nonselective beta blockers) was used.

A protocol for using and monitoring the AC therapy was conducted: in the first week, a loading dose of $200 \mathrm{U} /$ $\mathrm{kg}$ enoxaparin was administered to all patients; afterwards, AC with enoxaparin $(n=36)$ or VKA $(n=18)$ was used. Enoxaparin was preferred in patients at higher risk of bleeding: history of variceal bleeding, ongoing variceal band ligation surveillance program, thrombocytes < $50 \times 103 / \mathrm{mm} 3$ ) as well as for those who could not assess their international normalized ratio (INR) value periodically. Anticoagulant therapy was avoided in patients with history of variceal bleeding associated with severe coagulopathy (INR $\geq 2$ ) or severe thrombocytopenia.

During the study period, both groups were followed-up periodically. Patients on VKA have had their INR assessed weekly, with a target value of 2-2.5. Thrombus evolution (regression, progression, or stable PVT) was assessed by CT scan every 3 months. Luminal vascular patency was expressed as a percentage on CT scan and regression was considered for any increase in the lumen permeability compared to baseline. Furthermore, patients were asked at each appointment about acute or chronic bleeding events. The events that led to discontinuation of the AC therapy were bleeding with secondary anemia, liver transplantation and patient death. The mean time follow up period was 32 months (range: 3 -109) considered from diagnosing the PVT until the last contact (death, liver transplant, last follow-up visit).
Data were analyzed using IBM SPSS statistics version 20. For quantitative variables we estimated mean, range, standard deviation (SD) and median, and for categorical variables absolute and relative frequencies were calculated. Comparison between groups was performed using Welch T tests two-sided for continuous variables, and for categorical variables we used two-sided chi-square tests for independent proportion or Fisher's exact tests. Multivariate analysis was conducted by logistic regression and survival curves have been compared using log-rank test and cox regression. P-values less than 0.05 were considered statistically significant.

\section{RESULTS}

A total of 107 patients with liver cirrhosis and PVT were included in this study. The baseline characteristic are displayed in Table I.

Most patients had partial thrombosis with portal trunk and confluence involvement. The study cohort was divided into two separate groups, matched according to Child Pugh score, one receiving $\mathrm{AC}(\mathrm{n}=54)$ and the other not receiving it $(\mathrm{n}=53)$. Both groups showed similarities regarding gender, age and etiology of cirrhosis (Table I). Clinical and biological features in each group were observed: most patients in the AC group had a MELD score between 11-19 and were in an intermediate clinical condition (Child Pugh B) before the diagnosis of PVT. A grade one ascites was noted in more than $40 \%$ of patients in each group; nevertheless, a significant number of patients did not have ascites prior to the diagnosis of PVT (29.62\% in the AC group and $20.75 \%$ in the non-AC group). Six (11.11\%) patients in AC group and ten (18.86\%) in non-AC group were diagnosed with hepatic encephalopathy of varying degrees. History of spontaneous bacteria peritonitis was observed at $4(7.4 \%)$ patients in the AC group and at $6(11.32 \%)$ in the non-AC group $(\mathrm{p}=0.5)$.

A univariate binary simple logistic regression was conducted to assess whether $\mathrm{AC}$ is a predictor for thrombus regression. The model was significant $(\mathrm{OR}=2.43, \mathrm{p}=0.026$, 95\%CI: 1.11-6.167), anticoagulated patient being two times more likely to experience regression of the thrombus. Regression of portal vein thrombosis was found in 30 patients (55.6\%) in the AC group and in 18 (34\%) in the untreated group. This effect was observed after approximately 6 months of AC therapy (Table II).

A total of 98 patients $(91.58 \%)$ had varices (Table I). Thirtyfive (32.71\%) were treated endoscopically: 22 (20.56\%) from the AC group, and 13 (12.14\%) from the untreated group. Endoscopic treatment was performed for patients showing red cherry spots and grade II varices and for those with large varices (grade III or IV). Previous variceal bleeding was found in 18 patients $(33.3 \%)$ of patients in the AC group, respectively in $24(45.3 \%)$ in non-AC group ( $\mathrm{p}=0.3)$. Bleeding events were more frequent in the AC group, 10 cases of which 3 were mild (2 epistaxis, one oral bleeding) and 7 variceal bleeding that required hospitalization. The untreated group had only four cases of variceal bleeding (Table II). Of all patients with variceal bleeding 3 occurred after band ligation in the AC group and 2 in the untreated group. Two of these patients (one treated and one untreated) associated progression of the PVT. 
Table I. Patients' characteristics

\begin{tabular}{|c|c|c|c|}
\hline & $\begin{array}{l}\text { Anticoagulation group } \\
\qquad(\mathrm{n}=54)\end{array}$ & $\begin{array}{l}\text { Untreated group } \\
\qquad(\mathrm{n}=53)\end{array}$ & $\mathrm{p}$ \\
\hline Age & $53(23-73)$ & $55.65(25-75)$ & 0.20 \\
\hline Male, n (\%) & $29(53.7)$ & $25(47.1)$ & 0.44 \\
\hline $\begin{array}{c}\text { Etiology, n (\%) } \\
\text { HBV } \\
\text { HBV+HDV } \\
\text { HVC } \\
\text { Ethanolic } \\
\text { Cryptogenic } \\
\text { Autoimmune }\end{array}$ & $\begin{array}{c}9(16.7) \\
16(29.6) \\
10(18.5) \\
12(22.2) \\
5(9.3) \\
2(3.7)\end{array}$ & $\begin{array}{l}6(11.3) \\
15(28.3) \\
14(26.4) \\
11(20.8) \\
2(3.8) \\
5(9.4)\end{array}$ & 0.73 \\
\hline $\begin{array}{l}\text { MELD score at diagnosis, } \mathrm{n}(\%) \\
\quad<11 \\
\quad 11-19 \\
20-30\end{array}$ & $\begin{array}{c}9(16.7) \\
43(79.6) \\
2(3.7)\end{array}$ & $\begin{array}{l}10(18.9) \\
42(79.2) \\
1(1.8)\end{array}$ & 0.19 \\
\hline $\begin{array}{l}\text { Child Pugh score, n (\%) } \\
\text { A } \\
\text { B } \\
\text { C }\end{array}$ & $\begin{array}{l}13(24) \\
40(74) \\
1(2)\end{array}$ & $\begin{array}{l}14(26.4) \\
37(69.8) \\
2(3.8)\end{array}$ & 0.99 \\
\hline $\begin{array}{l}\text { Esophageal varices, } \mathrm{n}(\%) \\
\text { Grade } 1 \\
\text { Grade } 2 \\
\text { Grade } 3 \\
\text { Grade } 4 \\
\text { No varices }\end{array}$ & $\begin{array}{l}26(48.1) \\
21(38.9) \\
3(5.6) \\
1(1.9) \\
3(5.6)\end{array}$ & $\begin{array}{l}18(34) \\
20(37.7) \\
8(15.1) \\
1(1.9) \\
6(11.3)\end{array}$ & 0.31 \\
\hline $\begin{array}{l}\text { Ascites at diagnosis, } \mathrm{n}(\%) \\
\text { Grade } 1 \\
\text { Grade } 2 \\
\text { Grade } 3 \\
\text { No ascites }\end{array}$ & $\begin{array}{l}22(40.8) \\
8(14.8) \\
8(14.8) \\
16(29.6)\end{array}$ & $\begin{array}{l}25(47.2) \\
10(18.9) \\
7(13.2) \\
11(20.7)\end{array}$ & 0.70 \\
\hline HE before PVT & $6(11.1)$ & $10(18.9)$ & 0.49 \\
\hline SBP, n (\%) & $4(7.4)$ & $6(11.3)$ & 0.50 \\
\hline $\begin{array}{l}\text { Thrombus extension, } \mathrm{n}(\%) \\
\text { Brunch } \\
\text { Trunk } \\
\text { Confluent } \\
\text { Superior mesenteric vein thrombosis }\end{array}$ & $\begin{array}{l}28(51.9) \\
45(83.3) \\
33(61.1) \\
18(33.3)\end{array}$ & $\begin{array}{l}26(49.1) \\
35(66) \\
27(51) \\
20(37.7)\end{array}$ & $\begin{array}{l}0.46 \\
0.33 \\
0.19 \\
0.39\end{array}$ \\
\hline $\begin{array}{l}\text { Degree of portal vein obstruction, } \mathrm{n}(5) \\
\text { Complete } \\
\text { Partial }\end{array}$ & $\begin{array}{c}9(16.7) \\
45(83.3)\end{array}$ & $\begin{array}{l}13(24.5) \\
40(75.5)\end{array}$ & 0.26 \\
\hline $\begin{array}{l}\text { Platelet count, } \mathrm{n}(\%) \\
\quad>50 \times 10^{3} / \mathrm{mm}^{3} \\
\quad<50 \times 10^{3} / \mathrm{mm}^{3}\end{array}$ & $\begin{array}{l}32(59.3) \\
22(40.7)\end{array}$ & $\begin{array}{l}38(71.7) \\
15(28.3)\end{array}$ & 0.06 \\
\hline History of variceal bleeding, $\mathrm{n}(\%)$ & $18(33.3 \%)$ & $24(45.2)$ & 0.30 \\
\hline
\end{tabular}

HBV: hepatitis B virus; HDV: hepatitis D virus; HCV: hepatitis C virus; MELD: model for end stage liver disease; HE: hepatic encephalopathy; PVT: portal vein thrombosis; SBP: spontaneous bacterial peritonitis.

A simple univariate binary logistic regression was conducted to assess whether the MELD score, thrombocytes and $\mathrm{AC}$ were predictors of the bleeding events. Only one variable made a significant predictor for hemorrhagic complications: thrombocytes less than $50 \times 10^{3} / \mathrm{mm}^{3}$ rose the risk of bleeding events in these patients 8 times (Table III).

Table II. Outcome of patients with cirrhosis and portal vein thrombosis in the presence or absence of anticoagulant therapy

\begin{tabular}{lccccc}
\hline & $\begin{array}{c}\text { Anticoagulation } \\
\text { group }(\mathrm{n}=54)\end{array}$ & $\begin{array}{c}\text { Untreated group } \\
(\mathrm{n}=53)\end{array}$ & OR & $95 \% \mathrm{CI}$ & $\mathrm{p}$ \\
\hline Evolution of PVT, $\mathrm{n}(\%)$ & & & & & \\
$\quad$ Progression & $6(11.1)$ & $8(15.1)$ & 0.703 & $0.216-2.177$ & 0.543 \\
$\quad$ Regression & $30(55.6)$ & $18(34)$ & 2.430 & $1.11-6.167$ & 0.026 \\
$\quad$ Stationary & $18(33.3)$ & $27(50.9)$ & 0.566 & $0.262-1.255$ & 0.066 \\
Hemorrhagic complication $\mathrm{n}(\%)$ & $10(18.5)$ & $4(7.5)$ & 2.78 & $0.863-10.733$ & 0.102 \\
\hline
\end{tabular}

PVT: portal vein thrombosis; OR: odds ratio; CI: confidence interval. 
Table III. Logistic regression predicting hemorrhagic complications

\begin{tabular}{lccc}
\hline & OR & $95 \%$ CI & $\mathrm{p}$ \\
\hline Thrombocytes $<50 \times 10^{3} / \mathrm{mm}^{3}$ & 8.266 & $2.310-39.211$ & 0.002 \\
MELD score & 0.982 & $0.819-1.153$ & 0.838 \\
Anticoagulation therapy & 2.784 & $0.863-10.733$ & 0.102 \\
\hline
\end{tabular}

MELD: model for end stage liver disease; OR: odds ratio; CI: confidence interval.

Survival was better in the AC treatment group [21 patients $(39.6 \%)$ died in the untreated group vs 12 patients $(22.2 \%)$ in the AC treated group] and the difference was significant $(68.4 \%$ vs $48.7 \%$ at 5 years and $92.7 \%$ vs $77.8 \%$ at 1 year, $\mathrm{p}=0.038)$. In contrast to this finding, survival was lower in patients that experienced bleeding events $(37.2 \%$ survival at 5 years, mean time survival 44 months, $\mathrm{p}=0.008$ ) (Figs. 1 and 2). Median survival was 58 months in untreated group and double (106 months) in the treated group.

In order to assess how AC and bleeding influenced patients' survival, we performed a Cox regression demonstrating that patients without $\mathrm{AC}$ have higher mortality than patients on AC therapy $(\mathrm{HR}=2.14,95 \% \mathrm{CI}: 1.02-4.46, \mathrm{p}=0.042)$. Patients who had hemorrhagic events had a median survival of 41 months compared to 69 months in those without bleeding events. Furthermore, death was recorded in $57.1 \%$ of patients who experienced a bleeding event vs $26 \%$ of patients without bleeding events. Thus, patients with bleeding events had a significant higher mortality than patients without bleeding events $(\mathrm{HR}=2.79,95 \% \mathrm{CI}=1.25-6.23, \mathrm{p}=0.012)$.

To investigate how these parameters interact together, we divided the patients in 4 groups: group 1 - patients with AC and without bleeding events (with the best outcome), group 2 - patients without AC and without bleeding events, group 3 - patients with AC and with bleedings events and group 4 - patients without AC and with bleeding events (there were only 4 patients in this group, so the inferences regarding this

\section{Survival Curves Comparison}
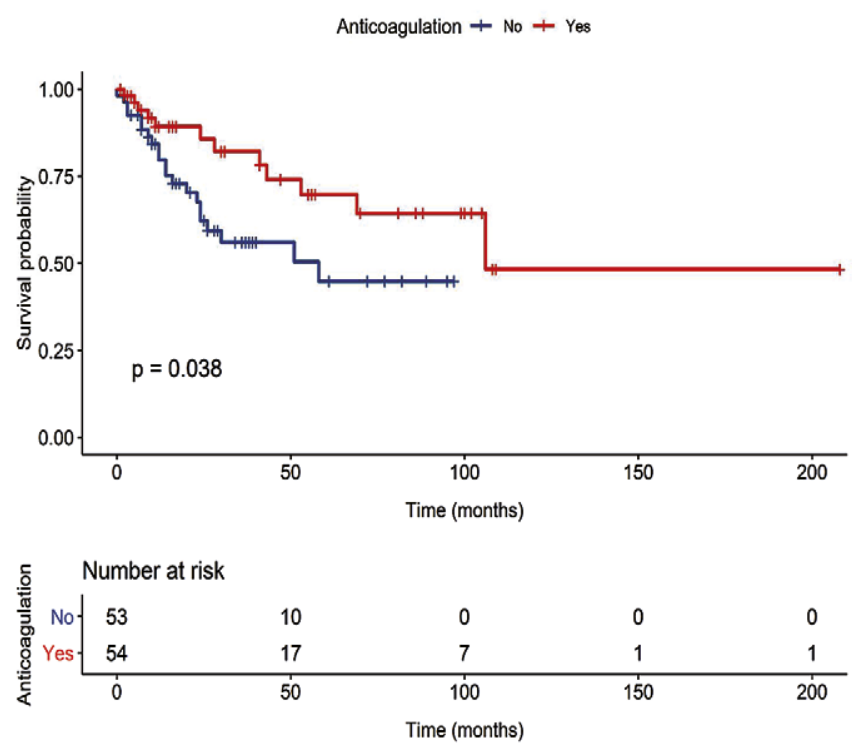

Fig. 1. Survival curves among patients with cirrhosis and PVT according to the use of anticoagulant therapy.

\section{Survival Curves Comparison}

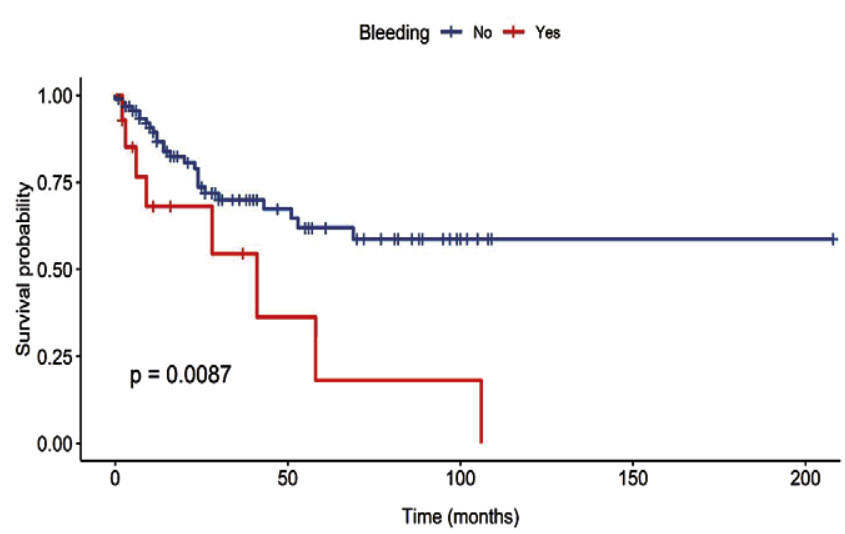

\section{Number at risk}

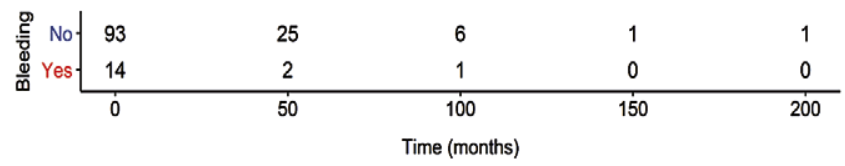

Fig. 2. Survival rates among patients with cirrhosis and portal vein thrombosis according to the hemorrhagic complications.

are not accurate). A simple Cox regression on this parameter, using group 1 as reference found that patients on AC therapy who associate one or more bleeding events have the lowest survival rate (Table IV).

Table IV. The influence of anticoagulation therapy and bleeding events on survival

\begin{tabular}{lccc}
\hline Parameters & Coefficient & $\mathrm{p}$ & HR [95\%CI] \\
\hline Group 1 & REFERENCE & - & - \\
Group 2 & 1.282 & 0.0068 & $3.60[1.42$ to 9.13] \\
Group 3 & 1.936 & 0.0009 & $6.93[2.20$ to 21.83] \\
Group 4 & 1.533 & 0.0651 & $4.63[0.92$ to 23.13] \\
\hline
\end{tabular}

Cox regression; Group 1 - patients with $\mathrm{AC}$ and without bleeding events; Group 2 - patients without AC and without bleeding events; Group 3 patients with AC and with bleedings events; group 4 - patients withoutAC and with bleeding events. HR: hazard ratio; CI: confidence interval.

\section{DISCUSSION}

Portal vein thrombosis represents a common consequence and sometimes a cause for worsening liver disease in advanced cirrhotic patients $[7,8]$. This group of patients tends to have cirrhosis decompensations, increased morbidity, and hospitalization, with poor overall survival. Moreover, bleeding events cannot be predicted, due to the presence of "rebalanced hemostasis", a hemostatic profile that defines the altered balance between pro- and anticoagulant factors [9]. In the last decade, many observational studies have highlighted the beneficial role of AC therapy in patients with PVT and cirrhosis $[7,8,10]$. However, there are still concerns regarding the safety and efficacy of anticoagulants among cirrhotic patients diagnosed with PVT. Therefore, it is important to define the features that will help select those patients that will mostly benefit from AC therapy.

In our study, the use of AC had a positive effect on PVT evolution; more than half in the AC group experienced 
regression of thrombosis (55.6\%). Interestingly, there was a minimum effect on the rate of progression of thrombosis in both groups ( $11.1 \%$ vs $15.1 \%)$, thus emphasizing the question whether all patients should be given AC considering that the risk of progression is low. These data match the data in the literature: in their meta-analysis Loffredo et al. [11] demonstrated that the use of AC is correlated with high regression (more than $50 \%$ experienced complete recanalization). Moreover, this phenomenon was accompanied by an insignificant risk of bleeding events and a low rate of liver disease progression. Another meta-analysis by Qi X et. al. [8] described similar findings, with a higher rate of recanalization (67\%) after AC therapy. An important aspect when using AC therapy in this set of patients is the timing: initiation of AC therapy should be done as soon as possible after the diagnosis of PVT. Studies have shown the beneficial role of AC when treatment was started within 6 months of the onset of PVT $[12,13]$. Unfortunately, PVT is rarely diagnosed in the early stages, being frequently clinically asymptomatic [14].

Our study's AC protocol consisted of a loading dose and a maintenance dose: LMWH in the loading phase followed by LMWH or VKA. Although more than $50 \%$ of patients who underwent $\mathrm{AC}$ treatment experienced PVT regression, a significant percentage of them (18.5\%) suffered a bleeding event (more than $60 \%$ of those undergoing hospitalization and cessation of AC therapy). This rate is higher than expected considering those cited in the general literature, consonant to data coming from other groups: even though in their metaanalysis Loffredo et al. [11] described the insignificant risk of bleeding events opposed to the higher rates of PVT regression, Kwon J et al. [15] reported a $14.3 \%$ rate of bleeding events in their study when using AC therapy in patients with cirrhosis and PVT. As in our study, the main risk factor for bleeding events was platelet level. The fact that in several studies such as of Delgado's et al. [12] and Villa's et al. [16], the use of AC in PVT has not been associated with a significant rate of bleeding events, might suggest that AC therapy alone may not be the only factor dictating the risk of complications.

Data regarding long term survival of patients with PVT are not clear. D'Amico et al. [17] found no correlation between the presence of PVT and 6-months mortality in patients with liver cirrhosis. Ferreira et al. [18] found a significant correlation between PVT and 3-year mortality, even if no differences were observed at one year follow-up. Overall mortality is thought to be around $60 \%$ [19]. Those data indicate that PVT is a slowly evolving process and AC therapy should be continued even after complete recanalization of the portal vein, at least in patients eligible for liver transplantation, and this is stated in current guidelines [20]. In this regard, our study showed an overall benefit of using AC therapy, positive data emerging after the first year of use.

\section{CONCLUSIONS}

In our cohort of cirrhotic patients with PVT, more than 50\% of patients receiving AC therapy showed response to therapy defined as regression of PVT. Most of them obtained partial recanalization. The bleeding complication rate was higher than expected, reaching $18 \%$. The overall mortality was lower in the AC group, confirming the existing data in the literature that showed a favorable effect on survival of $\mathrm{AC}$ in cirrhotic patients with PVT.

Conflicts of interest: None to declare.

Author's contribution: L.G. conceived and designed the study. M.F. collected the data and performed the statistical analysis. S.I. monitored the patients and performed the statistical analysis. C.E. collected the data. R.C, C.P monitored the patients. A.C., R.D., M.G., I.L. performed the imaging studies. All authors critically revised the manuscript, approved the final version to be published, and agree to be accountable for all aspects of the work.

\section{REFERENCES}

1. Khan AN, MacDonald S, Sheen AJ, Sherlock D, Al-Khattab Y. Portal Vein Thrombosis. [Last accessed on 2008 Jun 10]. Available from:http:// www.emedicine.com/radio/topic571.htm

2. Amitrano L, Guardascione MA, Menchise A, et al. Safety and efficacy of anticoagulation therapy with low molecular weight heparin for portal vein thrombosis in patients with liver cirrhosis. J Clin Gastroenterol 2010;44:448-451. doi:10.1097/MCG.0b013e3181b3ab44

3. Chung JW, Kim GH, Lee JH, et al. Safety, efficacy, and response predictors of anticoagulation for the treatment of nonmalignant portal-vein thrombosis in patients with cirrhosis: a propensity score matching analysis. Clin Mol Hepatol 2014;20:384-391. doi:10.3350/cmh.2014.20.4.384

4. Chen H, Liu L, Qi X, et al. Efficacy and safety of anticoagulation in more advanced portal vein thrombosis in patients with liver cirrhosis. Eur J Gastroenterol Hepatol 2016;28:82-89. doi:10.1097/ MEG.0000000000000482

5. Lisman T, Porte RJ. Rebalanced hemostasis in patients with liver disease: evidence and clinical consequences. Blood 2010;116:878-885. doi:10.1182/blood-2010-02-261891

6. Wang L, Guo X, Xu X, et al. Anticoagulation Favors Thrombus Recanalization and Survival in Patients With Liver Cirrhosis and Portal Vein Thrombosis: Results of a Meta-Analysis. Adv Ther 2021;38:495520. doi:10.1007/s12325-020-01550-4

7. Mannucci PM, Tripodi A. Direct oral anticoagulants and cirrhosis: More evidence still needed for efficacy and safety in portal vein thrombosis. Vascul Pharmacol 2019;113:92-93. doi:10.1016/j.vph.2018.06.005

8. Qi X, De Stefano V, Li H, Dai J, Guo X, Fan D. Anticoagulation for the treatment of portal vein thrombosis in liver cirrhosis: A systematic review and meta-analysis of observational studies. Eur J Intern Med 2015;26:23-29. doi:10.1016/j.ejim.2014.12.002

9. nder the auspices of the Italian Association for the Study of Liver Diseases (AISF) and the Italian Society of Internal Medicine (SIMI). Hemostatic balance in patients with liver cirrhosis: Report of a consensus conference. Dig Liver Dis 2016;48:455-467. doi:10.1016/j.dld.2016.02.008

10. La Mura V, Braham S, Tosetti G, et al. Harmful and Beneficial Effects of Anti-coagulants in Patients With Cirrhosis and Portal Vein Thrombosis. Clin Gastroenterol Hepatol 2018;16:1146-1152.e4. doi:10.1016/j. cgh.2017.10.016

11. Loffredo L, Pastori D, Farcomeni A, Violi F. Effects of Anticoagulants in Patients With Cirrhosis and Portal Vein Thrombosis: A Systematic Review and Meta-analysis. Gastroenterology 2017;153:480-487.e1. doi:10.1053/j.gastro.2017.04.042 
12. Delgado MG, Seijo S, Yepes I, et al. Efficacy and safety of anticoagulation on patients with cirrhosis and portal vein thrombosis. Clin Gastroenterol Hepatol 2012;10:776-783. doi:10.1016/j.cgh.2012.01.012

13. Rodriguez-Castro KI, Vitale A, Fadin M, et al. A prediction model for successful anticoagulation in cirrhotic portal vein thrombosis. Eur J Gastroenterol Hepatol 2019;31:34-42. doi:10.1097/ MEG.0000000000001237

14. Senzolo M, Ferronato C, Burra P, Sartori MT. Anticoagulation for portal vein thrombosis in cirrhotic patients should be always considered. Intern Emerg Med 2009;4:161-162. doi:10.1007/s11739-008-0219-y

15. Kwon J, Koh Y, Yu SJ, Yoon JH. Low-molecular-weight heparin treatment for portal vein thrombosis in liver cirrhosis: Efficacy and the risk of hemorrhagic complications. Thromb Res 2018;163:71-76. doi: 10.1016/j. thromres.2018.01.032

16. Villa E, Camma C, Marietta M, et al. Enoxaparin prevents portal vein thrombosis and liver decompensation in patients with advanced cirrhosis. Gastroenterology 2012;143:1253-1260.e4. doi:10.1053/j. gastro.2012.07.018

17. D’Amico G, De Franchis R; Cooperative Study Group. Upper digestive bleeding in cirrhosis. Post-therapeutic outcome and prognostic indicators. Hepatology 2003;38:599-612. doi:10.1053/jhep.2003.50385

18. Ferreira CN, Rodrigues T, Alexandrino P, Ramalho F, Velosa JF. 1571 Portal vein thrombosis in cirrhotic patients is associated with advanced liver disease and predicts poor longterm prognosis. Hepatology 2010;52 (S1):1072A. doi:10.1002/hep.23995

19. Qi X, Dai J, Yang M, Ren W, Jia J, Guo X. Association between Portal Vein Thrombosis and Survival in Non-Liver-Transplant Patients with Liver Cirrhosis: A Systematic Review of the Literature. Gastroenterol Res Pract 2015;2015:480842. doi:10.1155/2015/480842

20. European Association for the Study of the Liver. EASL Clinical Practice Guidelines: Vascular diseases of the liver. J Hepatol 2016;64:179-202. doi:10.1016/j.jhep.2015.07.040 